MITSUBISHI ELECTRIC RESEARCH LABORATORIES

http://www.merl.com

\title{
On a Ratio of Functions of Exponential Random Variables and Some Applications
}

\author{
Ramesh Annavajjala, A. Chockalingam, Saif Mohammed
}

TR2010-118 December 2010

\begin{abstract}
Consider L independent and identically distributed exponential random variables (r.vs) X1,X2,..,XL, and positive scalars b1,b2,..,bL. In this letter, we present the probability density function (pdf), cumulative distribution function and the Laplace transform of the pdf of the composite r.v $\mathrm{Z}=$ $(\mathrm{X} 1+\mathrm{X} 2+\ldots+\mathrm{XL}) 2 /(\mathrm{b} 1 * \mathrm{X} 1+\mathrm{b} 2 * \mathrm{X} 2+\ldots+\mathrm{bL} * \mathrm{XL})$. We show that the r.v $\mathrm{Z}$ appears in various communication systems such as 1) maximal ratio combining of signals received over multiple channels with mismatched noise variances, 2) M-ary phase-shift keying with spatial diversity and imperfect channel estimation, and 3) coded multi-carrier code-division multiple access reception affected by an unknown narrow-band interference, and the statistics of the r.v Z derived here enable us to carry out the performance analysis of such systems in closed-form.
\end{abstract}

IEEE Transactions on Communications

This work may not be copied or reproduced in whole or in part for any commercial purpose. Permission to copy in whole or in part without payment of fee is granted for nonprofit educational and research purposes provided that all such whole or partial copies include the following: a notice that such copying is by permission of Mitsubishi Electric Research Laboratories, Inc.; an acknowledgment of the authors and individual contributions to the work; and all applicable portions of the copyright notice. Copying, reproduction, or republishing for any other purpose shall require a license with payment of fee to Mitsubishi Electric Research Laboratories, Inc. All rights reserved.

Copyright (C) Mitsubishi Electric Research Laboratories, Inc., 2010

201 Broadway, Cambridge, Massachusetts 02139 



\title{
On a Ratio of Functions of Exponential Random Variables and Some Applications
}

\author{
Ramesh Annavajjala, Senior Member, IEEE, A. Chockalingam, Senior Member, IEEE, and \\ Saif. K. Mohammed, Student Member, IEEE
}

\begin{abstract}
Consider $L$ independent and identically distributed exponential random variables (r.vs) $X_{1}, X_{2}, \ldots, X_{L}$ and positive scalars $b_{1}, b_{2}, \ldots, b_{L}$. In this letter, we present the probability density function (pdf), cumulative distribution function and the Laplace transform of the pdf of the composite r.v $Z=$ $\left(\sum_{j=1}^{L} X_{j}\right)^{2} /\left(\sum_{j=1}^{L} b_{j} X_{j}\right)$. We show that the r.v $Z$ appears in various communication systems such as $i$ ) maximal ratio combining of signals received over multiple channels with mismatched noise variances, $i i) M$-ary phase-shift keying with spatial diversity and imperfect channel estimation, and iii) coded multi-carrier code-division multiple access reception affected by an unknown narrow-band interference, and the statistics of the r.v $Z$ derived here enable us to carry out the performance analysis of such systems in closed-form.
\end{abstract}

Index Terms-Exponential random variables, distribution of ratio of two random variables, bivariate Laplace transform, mismatched statistics, partial-band interference.

\section{INTRODUCTION}

Consider $L$ independent and identically distributed (i.i.d) exponential random variables (r.vs) $X_{1}, X_{2}, \ldots, X_{L}$, and $L$ positive numbers $b_{1}, b_{2}, \ldots, b_{L}$. In this letter, we are interested in the statistical properties of the r.v $Z$ defined as

$$
Z=\frac{\left(\sum_{j=1}^{L} X_{j}\right)^{2}}{\sum_{j=1}^{L} b_{j} X_{j}} .
$$

In particular, we are interested in the probability density function (pdf), cumulative distribution function (cdf) and the Laplace transform (LT) of the pdf (or simply, LT) of $Z$ in (1). Interestingly, in Section III, we show that the r.v $Z$ in (1) appears in various communication systems such as $i$ ) maximal ratio combining (MRC) of signals received over multiple channels with mismatched noise variances, ii) $M$-ary phaseshift keying (PSK) with spatial diversity and imperfect channel estimation, and $\mathrm{iii}$ ) coded multi-carrier code-division multiple access (MC-CDMA) reception affected by an unknown narrowband interference (NBI). Consequently, the statistics of the r.v $Z$ derived here enable us to carry out the performance analysis of such systems in closed-form.

Ramesh Annavajjala (ramesh.annavajjala@gmail.com) is with the Mitsubishi Electric Research Laboratories (MERL), Cambridge, MA, USA.

A. Chockalingam (achockal@ece.iisc.ernet.in) and Saif K. Mohammed (saifind2007@yahoo.com) are with the Electrical Communication Engineering department at the Indian Institute of Science, Bangalore, INDIA.

This work was conceived, and completed, long before the first author started working at MERL.
Note that, when $b_{i}=b$, for $i=1, \ldots, L, Z$ in (1) reduces to

$$
Z=\frac{1}{b} \sum_{j=1}^{L} X_{j} .
$$

That is, $Z$ is a sum of $L$ i.i.d exponential r.vs each with mean $1 / b$. The pdf, cdf and LT of $Z$ are well-known and are given by [1]

$$
\begin{aligned}
f_{Z}(z) & =\frac{e^{-z b} z^{L-1} b^{L}}{\Gamma(L)} \\
F_{Z}(z) & =\int_{0}^{z} f_{Z}(u) d u=1-e^{-b z} \sum_{n=0}^{L-1} \frac{b^{n} z^{n}}{n !} \\
\text { and } \mathcal{L}_{Z}(s) & =E\left[e^{-s Z}\right]=\left(\frac{b}{s+b}\right)^{L}
\end{aligned}
$$

where $\Gamma(n)$ is the standard Gamma function [2]. Except for the above case of equal $b_{i}$ 's, to the best of our knowledge, expressions for the pdf, cdf and LT of $Z$ for arbitrary positive values of $b_{i}$ 's, and for an arbitrary $L$, do not seem to be available in the literature. However, when $X_{i}$ 's are independent and non-identically distributed exponential r.vs with distinct means [3] derives the cdf, pdf and the LT of (1) with $L=2$. On the other hand, with the following two assumptions

- The mean values $E\left[X_{i}\right]$ are distinct

- For $j \neq i, b_{j} \neq b_{i}$ and $b_{j} E\left[X_{j}\right] \neq b_{i} E\left[X_{i}\right]$

[4, Appendix-A] presents only the cdf of $Z$ in (1) for an arbitrary value of $L$. It is important to note that when all the r.vs $X_{i}$ 's have identical means, as considered here, the cdf expression in [4, Appendix-A] is not applicable.

The rest of this letter is structured as follows. We present our main results on the statistical properties of the r.v $Z$ in Section II, and some example applications are considered in Section III. Numerical and simulation results are provided in Section IV. We conclude this paper in Section V.

\section{MAin Results}

In this section, we present our key contributions. Due to page-length limitations, we provide here only the final results in a self-contained fashion. The details are available in [5]. For clarity, we consider two cases: $i$ ) distinct values of $b_{i}$ 's appearing in (1) and $i i$ ) repeated occurrence of some of the $b_{i}$ 's in (1). 


$$
\begin{aligned}
F_{Z}(z)= & \sum_{j=1}^{L} \frac{\lambda_{j}}{b_{j} \Gamma(L-1)} \sum_{k=0}^{L-2}(-1)^{L-2-k}\left(\begin{array}{c}
L-2 \\
k
\end{array}\right) \Gamma(k+1) \sum_{l=0}^{k} \frac{1}{l !} \times \\
& \left\{b_{j} \Gamma(L-1-k+l)\left[1-e^{-b_{j} z} \sum_{m=0}^{L-2-k+l} \frac{\left(b_{j} z\right)^{m}}{m !}\right]-\right. \\
& \left.\frac{2 \Gamma(l+2(L-2-k)+2)}{b_{j}^{L-2-k} z^{L-1-k}}\left[1-e^{-b_{j} z} \sum_{p=0}^{l+2(L-2-k)+1} \frac{\left(b_{j} z\right)^{p}}{p !}\right]\right\}, \\
f_{Z}(z)= & \sum_{j=1}^{L} \frac{\lambda_{j}}{b_{j} \Gamma(L-1)} \sum_{k=0}^{L-2}\left(\begin{array}{c}
L-2 \\
k
\end{array}\right) \frac{(-1)^{k} \Gamma(L+k+1)}{z^{k+2} b_{j}^{k}}\left(1-e^{-b_{j} z} \sum_{l=0}^{L+k} \frac{b_{j}^{l} z^{l}}{l !}\right), \\
\text { and } \mathcal{L}_{Z}(s)= & \sum_{j=1}^{L} \frac{\lambda_{j}}{b_{j} \Gamma(L-1)} \sum_{k=0}^{L-2}\left(\begin{array}{c}
L-2 \\
k
\end{array}\right)(-1)^{k} b_{j} \Gamma(L)\left(\frac{b_{j}}{s}\right)^{L} \\
& \times \frac{{ }_{2} F_{1}\left(L, L+k+1 ; L+k+2 ;-\frac{b_{j}}{s}\right)}{L+k+1}, \operatorname{Real}\{s\}>0 .
\end{aligned}
$$

\section{A. Distinct Values of $b_{i}$ 's}

With $b_{i} \neq b_{j} \forall i \neq j$, the cdf, pdf and LT of pdf of $Z$ in (1) are respectively given by (6), (7), and (8), shown at the top of this page, where $\lambda_{j}=\prod_{i=1, i \neq j}^{L} \frac{b_{j}}{b_{j}-b_{i}}$ in (6)-(8), and ${ }_{2} F_{1}(\cdot, \cdot ; \cdot ; \cdot)$ is the hypergeometric function [2]. The proofs of (6)-(8) are presented in [5, Appendix-A].

\section{B. Repeated Occurrences of $b_{i}$ 's}

Assume that there are $N$ distinct values of $b_{i}$ (i.e., $\left.b_{1}, \ldots, b_{N}\right)$ with $0<b_{1}<b_{2}<\ldots<b_{N}$. Let $b_{i}$ occurs $n_{i}$ times so that we have $L=\sum_{i=1}^{N} n_{i}$. Then, the cdf, pdf and LT of pdf of $Z$ in (1) are respectively given by (9), (10), and (11), shown in the next page, where $\mu(i, j)$ in (9)-(11) is defined as

$$
\begin{aligned}
\mu(i, j)= & \frac{1}{b_{i}^{n_{i}-j}\left(n_{i}-j\right) !} \\
& \times \sum_{l_{1} \geq 0} \cdots \sum_{l_{i-1} \geq 0} \sum_{l_{i+1} \geq 0} \cdots \sum_{l_{N} \geq 0} \\
& \left(\begin{array}{l}
n_{i}-j \\
l_{1}, \ldots, l_{i-1}, l_{i+1}, \ldots, l_{N}
\end{array}\right) \prod_{j=1, j \neq i}^{N}(-1)^{l_{j}} \\
& \times \frac{\Gamma\left(n_{j}+l_{j}\right)}{\Gamma\left(n_{j}\right)}\left(\frac{b_{i}}{b_{i}-b_{j}}\right)^{n_{j}+l_{j}} b_{j}^{l_{j}} .
\end{aligned}
$$

The proofs of (9)-(11) are presented in [5, Appendix-B]. As a quick sanity check, with $n_{j}=1$ we have $N=L$, and it is easy to show that (9), (10) and (11) reduce to (6), (7) and (8), respectively.

\section{APPLICATIONS}

\section{A. Example 1: Noise Variance Mismatch on MRC Receiver Performance}

In the first example, we consider a spatial diversity system with $L$ receiver antennas operating over i.i.d Rayleigh fading channels. The complex-valued baseband received signal on the $l$ th branch is

$$
r_{l}=g_{l} X+n_{l}, \quad l=1, \ldots, L,
$$

where $X$ is the transmitted symbol with $E\left[|X|^{2}\right]=E_{s}$, where $E_{s}$ denote the average transmitted symbol energy, $g_{l}$ is a zeromean complex Gaussian r.v (CGRV) whose amplitude $\beta_{l} \triangleq$ $\left|g_{l}\right|^{2}$ has the pdf $f_{\beta_{l}}(x)=e^{-x}, x \geq 0$, and $n_{l}$ is a zero-mean $\mathrm{CG}$ noise r.v added at the receiver front end. We assume that $E\left[\left|n_{l}\right|^{2}\right]=N_{l}$. The instantaneous SNR on the $l$ th branch is denoted by $\gamma_{l}$, and is given by $E_{s} \beta_{l} / N_{l}$. The mean of $\gamma_{l}$ is $\bar{\gamma}_{l}=E_{s} / N_{l}$.

The output of a general linear diversity combiner with weights $\left(w_{l}, w_{2}, \ldots, w_{L}\right)$, where $w_{l}$ is the complex-valued weight applied on the $l$ th branch, is

$$
r=\sum_{l=1}^{L} w_{l} r_{l}=X \sum_{l=1}^{L} w_{l} g_{l}+\eta,
$$

where $\eta$ is zero-mean CGRV with conditional variance $E\left[|\eta|^{2}\right]=\sum_{l=1}^{L}\left|w_{l}\right|^{2} N_{l}$. With ideal MRC reception, we require $w_{l}=g_{l}^{*} / N_{l}$ [6]. Using these weights in (14) leads to

$$
r=X \sum_{l=1}^{L} \frac{\beta_{l}}{N_{l}}+\eta,
$$

where, conditioned on $\beta_{1}, \ldots, \beta_{N}, E\left[\eta \mid \beta_{1}, \ldots, \beta_{N}\right]=0$ and $E\left[|\eta|^{2} \mid \beta_{1}, \ldots, \beta_{N}\right]=\sum_{l=1}^{L} \beta_{l} / N_{l}$. The instantaneous output SNR of (15) is

$$
\begin{aligned}
\gamma_{\text {ideal-MRC }} & =\frac{E\left[\left|X \sum_{l=1}^{L} \frac{\beta_{l}}{N_{l}}\right|^{2} \mid \beta_{1}, \ldots, \beta_{N}\right]}{E\left[|\eta|^{2} \mid \beta_{1}, \ldots, \beta_{N}\right]} \\
& =\sum_{l=1}^{L} \frac{E_{s} \beta_{l}}{N_{l}}=\sum_{l=1}^{L} \gamma_{l},
\end{aligned}
$$

establishing the well-known fact that the output SNR of an ideal MRC receiver is equal to the sum of the individual branch 


$$
\begin{aligned}
& F_{Z}(z)=\sum_{i=1}^{N} \sum_{j=1}^{n_{i}} \frac{\mu(i, j)}{b_{i}^{j} \Gamma(j) \Gamma(L-j)} \sum_{p=0}^{L-j-1} \sum_{l=0}^{p} \frac{\Gamma(p+1)}{l !} \frac{(-1)^{L-j-1-p}}{b_{i}^{1-j}}\left(\begin{array}{c}
L-j-1 \\
p
\end{array}\right) \\
& \times\left\{b_{i} \Gamma(L-1-p+l)\left\{1-e^{-b_{i} z} \sum_{q=0}^{L-2-p+l} \frac{\left(b_{i} z\right)^{q}}{q !}\right\}-\right. \\
& \left.\frac{2 \Gamma(2(L-2-p)+l+2)}{z^{(L-1-p)} b_{i}^{L-2-p}}\left\{1-e^{-b_{i} z} \sum_{r=0}^{2(L-2-p)+l+1} \frac{\left(b_{i} z\right)^{r}}{r !}\right\}\right\}, \\
& f_{Z}(z)=\sum_{i=1}^{N} \sum_{j=1}^{n_{i}} \frac{\mu(i, j)}{b_{i}^{j} \Gamma(j) \Gamma(L-j)} \sum_{k=0}^{L-j-1}\left(\begin{array}{c}
L-j-1 \\
k
\end{array}\right) \frac{(-1)^{k} \Gamma(L+j+k)}{b_{i}^{k} z^{k+j+1}} \\
& \times\left(1-e^{-b_{i} z} \sum_{l=0}^{L+j+k-1} \frac{b_{i}^{l} z^{l}}{l !}\right) \\
& \text { and } \mathcal{L}_{Z}(s)=\sum_{i=1}^{N} \sum_{j=1}^{n_{i}} \frac{\mu(i, j)}{b_{i}^{j} \Gamma(j) \Gamma(L-j)} \sum_{k=0}^{L-j-1}\left(\begin{array}{c}
L-j-1 \\
k
\end{array}\right)(-1)^{k} b_{i}^{j} \Gamma(L)\left(\frac{b_{i}}{s}\right)^{L} \\
& \times \frac{{ }_{2} F_{1}\left(L, L+k+j ; L+k+j+1 ;-\frac{b_{i}}{s}\right)}{L+k+j}, \quad \operatorname{Real}\{s\}>0 .
\end{aligned}
$$

SNRs [6]. When the receiver does not have the knowledge of $N_{l}, l=1, \ldots, L$, it simply uses $w_{l}=g_{l}^{*}$, leading to

$$
r=X \sum_{l=1}^{L} \beta_{l}+\eta
$$

where now $E\left[|\eta|^{2} \mid \beta_{1}, \ldots, \beta_{N}\right]=\sum_{l=1}^{L} \beta_{l} N_{l}$. The output SNR of this non-ideal MRC receiver is

$$
\gamma_{\text {nonideal-MRC }}=\frac{E_{s}\left[\sum_{l=1}^{L} \beta_{l}\right]^{2}}{\sum_{l=1}^{L} N_{l} \beta_{l}}=\frac{\left[\sum_{l=1}^{L} \beta_{l}\right]^{2}}{\sum_{l=1}^{L} \frac{\beta_{l}}{\bar{\gamma}_{l}}} .
$$

Using the Cauchy-Schwartz inequality, it can be readily shown that $\gamma_{\text {nonideal-MRC }} \leq \gamma_{\text {ideal-MRC }}$, and the equality holds if and only if $N_{l}=N, \forall l=1, \ldots, L$. Upon noticing that (18) is identical to (1) with $b_{i}=1 / \bar{\gamma}_{i}, i=1, \ldots, L$, one can compute the outage probability of received SNR, $P_{\text {out }}=\operatorname{Prob}\left(\gamma_{\text {nonideal-MRC }}<\gamma_{T}\right)=\int_{0}^{\gamma_{T}} f_{\gamma_{\text {nonideal-MRC }}}(x) d x$, where $\gamma_{T}$ is a pre-determined SNR threshold, in closed-form, using (6) or (7) ((9) or (10)) for distinct values of $\bar{\gamma}_{i}$ (for repeated values of $\left.\bar{\gamma}_{i}\right)$. In a similar manner, the average received SNR, $\bar{\gamma}_{\text {nonideal-MRC }}=E\left[\gamma_{\text {nonideal-MRC }}\right]=-\left.\frac{d}{d s} \mathcal{L}_{\gamma_{\text {nonideal-MRC }}}(s)\right|_{s=0}$, as well as the moment-generating function-based [1] average error probability of various modulation schemes can be obtained by either (8) or (11) (depending upon whether $\bar{\gamma}_{i}$ 's are distinct are not).

\section{B. Example 2: M-PSK Receiver Performance with Imperfect CSI}

Here, we study the performance of coherent $M$-PSK modulation on independent and non-identically distributed (i.n.d) Rayleigh fading channels with receive diversity and imperfect channel state information (CSI). The low-pass equivalent baseband received signal on the $l$ th branch is

$$
r_{l}=g_{l} X+n_{l},
$$

where $X$ belongs to the $M$-PSK constellation with an average energy $E\left[|X|^{2}\right]=E_{s}, g_{l}$ is a zero-mean CGRV with $E\left[\left|g_{l}\right|^{2}\right]=\Omega_{l}$, and the noise $n_{l}$ is a zero-mean CGRV with variance $N_{0}$. Assuming a linear channel estimation process (i.e., the channel estimate is obtained as a linear combination of received known (or pilot) symbols), we model the true and estimated channel gains on each branch by a bi-variate complexGaussian distribution. This assumption is satisfied by a variety of channel estimation schemes such as minimum mean-squareerror (MMSE) and pilot symbol assisted modulation (PSAM) based channel estimation schemes [7]. Let $p_{l}$, a zero-mean CGRV, denote the channel estimate on the $l$ th branch with $E\left[\left|p_{l}\right|^{2}\right]=\Lambda_{l}$. Then, by making use of the assumption that $p_{l}$ and $g_{l}$ are jointly Gaussian, we can write $g_{l}$ in terms of $p_{l}$ as [8]

$$
g_{l}=\rho_{l} \sqrt{\frac{\Omega_{l}}{\Lambda_{l}}} p_{l}+\sqrt{\left(1-\rho_{l}^{2}\right) \Omega_{l}} v_{l},
$$

where $v_{l}$ is a zero-mean CGRV, independent of $p_{l}$, with $E\left[\left|v_{l}\right|^{2}\right]=1$, and $\rho_{l}=E\left[g_{l} p_{l}^{*}\right] / \sqrt{E\left[\left|p_{l}\right|^{2}\right] E\left[\left|g_{l}\right|^{2}\right]}$. Here, we assume that $\rho_{l}$ is real with $\rho_{l}>0, \forall l=1, \ldots, L$, which is satisfied by MMSE and PSAM-based channel estimation models. Using (20) in (19), we have

$$
\begin{aligned}
r_{l} & =X p_{l} \rho_{l} \sqrt{\frac{\Omega_{l}}{\Lambda_{l}}}+X \sqrt{\left(1-\rho_{l}^{2}\right) \Omega_{l}} v_{l}+n_{l} \\
& =X p_{l} \rho_{l} \sqrt{\frac{\Omega_{l}}{\Lambda_{l}}}+\eta_{l},
\end{aligned}
$$

where $\eta_{l}$ is a zero-mean CGRV with variance $E[|\eta|]^{2}=N_{0}+$ $E_{s}\left(1-\rho_{l}^{2}\right) \Omega_{l}$. The combiner output is

$$
r=\sum_{l=1}^{L} p_{l}^{*} r_{l}=X \sum_{l=1}^{L}\left|p_{l}\right|^{2} \rho_{l} \sqrt{\frac{\Omega_{l}}{\Lambda_{l}}}+\sum_{l=1}^{L} \eta_{l} p_{l}
$$


The output SNR is then given by

$$
\gamma_{\mathrm{M}-\mathrm{PSK}}=\frac{E_{s}\left[\sum_{l=1}^{L}\left|p_{l}\right|^{2} \rho_{l} \sqrt{\frac{\Omega_{l}}{\Lambda_{l}}}\right]^{2}}{\sum_{l=1}^{L}\left|p_{l}\right|^{2}\left(N_{0}+E_{s}\left(1-\left|\rho_{l}\right|^{2}\right) \Omega_{l}\right)} .
$$

Upon letting $Z_{l}=\left|p_{l}\right|^{2} \rho_{l} \sqrt{\frac{\Omega_{l}}{\Lambda_{l}}}$, where $Z_{l}$ now is exponentially distributed with mean $\bar{Z}_{l}=\rho_{l} \sqrt{\Omega_{l} \Lambda_{l}}$, (23) can be conveniently written as

$$
\gamma_{\mathrm{M}-\mathrm{PSK}}=\frac{\left[\sum_{l=1}^{L} Z_{l}\right]^{2}}{\sum_{l=1}^{L} Z_{l} b_{l}}
$$

where

$$
b_{l}=\left(\frac{N_{0}+E_{s}\left(1-\left|\rho_{l}\right|^{2}\right) \Omega_{l}}{E_{s}}\right) \sqrt{\frac{\Lambda_{l}}{\Omega_{l}}} \frac{1}{\rho_{l}} .
$$

With $\rho_{l} \sqrt{\Omega_{l} \Lambda_{l}}=\mathrm{C}, \quad l=1, \ldots, L$, where $\mathrm{C}$ is a constant that does not depend on the branch index $l$, the outage probability, the average received SNR of $\gamma_{\mathrm{M} \text {-PSK }}$ in (24) and the average symbol error rate, $P_{s}=$ $(1 / \pi) \int_{0}^{(M-1) \pi / M} \mathcal{L}_{\gamma_{\text {M-PSK }}}\left(\sin ^{2}(\pi / M) / \sin ^{2} \theta\right) d \theta$, can be readily evaluated with the help of (6)-(11), derived in Section II.

\section{Example 3: Coded Multi-carrier CDMA System with Un- known Partial-Band Interference}

This example is concerned with the performance of a coded multi-carrier DS-CDMA (or, simply, MC-CDMA) system affected by a partial-band interference (PBI), as studied in [9] and [10]. Unlike [10], we assume that the receiver does not have the knowledge of the jammer side information, and exploit (6)(11), derived in Section II-A, to evaluate this system performance in closed-form. For completeness, we now summarize the system and the channel model from [9] and [10].

In a $K_{u}$-user uplink MC-CDMA system, the information bit sequence of the $k$ th user is denoted by $\left\{b_{n}^{(k)}\right\}$, where the subscript $n$ denotes the time index. Each bit $b_{n}^{(k)}$ is encoded by a channel code of rate $r_{c}$, and the resulting code symbols are interleaved. An ideal interleaver is assumed for the purpose of analysis. Each code symbol $d_{n}^{(k)}$ is then spread, binary phase modulated and transmitted over the $L$ disjoint frequency bands, each of width $W_{1}$. An optional symbol mapper is employed in [9] to perform coding across the sub-carriers. If $T_{c}$ and $W$, respectively, denote the chip duration and system bandwidth of a comparable single carrier (SC) CDMA system, then we have $W=(1+\beta) / T_{c}$, where $\beta \in(0,1]$ is the roll-off factor of the chip wave-shaping filter. The bandwidth available per sub-carrier in a MC-CDMA system is then $W_{1}=W / L=(1+\beta) /\left(T_{c} L\right)=(1+\beta) / T_{c_{1}}$, where $T_{c_{1}}=L T_{c}$ is the corresponding chip duration in the MC-CDMA system.

Mathematically, the signal at the output of the $k$ th user's transmitter can be written as

$$
\begin{aligned}
S_{k}(t)= & \sqrt{2 E_{c}} \sum_{n=-\infty}^{\infty} d_{\lfloor n / N\rfloor}^{(k)} c_{n}^{(k)} h\left(t-n L T_{c}\right) \\
& \times \sum_{m=1}^{L} \cos \left(2 \pi f_{m} t+\theta_{m}^{(k)}\right),
\end{aligned}
$$

where $\lfloor x\rfloor$ is the largest integer that is less than or equal to $x,\left\{c_{n}^{(k)}\right\}$ denotes the spreading sequence, $f_{m}$ is the center frequency (in Hertz) of the $m$ th sub-carrier, $\theta_{m}^{(k)}$ denotes the initial phase angle of the $k$ th user's $m$ th sub-carrier, $N$ is the number of chips per code symbol per sub-carrier, and $E_{c}$ denotes the energy per chip. Also, $h(t)$ denotes the impulse response of the chip wave-shaping filter. Here, we assume that $X(f)=|H(f)|^{2}$ satisfies the Nyquist criterion, where $H(f)$ is the Fourier transform of $h(t)$. Denoting by $\mathcal{S}_{F}$ the spreading factor associated with SC-CDMA, we have $\mathcal{S}_{F}=T_{s} / T_{c}=L T_{s} / T_{c_{1}}=L N$, where $T_{s}$ is the code symbol duration. With this, we can express $N$ as $N=\mathcal{S}_{F} / L$.

We assume that the channel is frequency-selective over the bandwidth $W$. However, the total bandwidth $W$ is assumed to be partitioned into $L$ disjoint frequency bands in such a way that each of these $L$ bands experiences independent, frequencyflat fading. In [11], conditions were derived for satisfying this assumption. With this, the received signal of the $k$ th user can be written as

$$
\begin{aligned}
r(t)= & \sum_{k=1}^{K_{u}} \sqrt{2 E_{c}} \sum_{n=-\infty}^{\infty} d_{\lfloor n / N\rfloor}^{(k)} c_{n}^{(k)} h\left(t-n L T_{c}-\tau_{k}\right) \\
& \times \sum_{m=1}^{L} \alpha_{m}^{(k)} \cos \left(2 \pi f_{m} t+\psi_{k, m}\right) \\
& +n_{W}(t)+n_{J}(t)
\end{aligned}
$$

where $\tau_{k}$ is the random time delay corresponding to the $k$ th user, assumed to be uniformly distributed in $\left[0, L T_{c}\right), K_{u}$ is the total number of active users in the system, $\alpha_{m}^{(k)}$ denotes the fade amplitude, $\phi_{m}^{(k)}$ denotes the random phase on the $m$ th subcarrier of the $k$ th user, and $\psi_{m}^{(k)}=\theta_{m}^{(k)}+\phi_{m}^{(k)}$ is the resultant phase on the $m$ th sub-carrier. The term $n_{W}(t)$ denotes the additive white Gaussian noise (AWGN) with a two-sided PSD of $\eta_{0} / 2$, whereas $n_{J}(t)$ represents Gaussian distributed PBI with a PSD of $S_{J}(f)$.

For the sake of analysis, we assume that the fades are independent across the users, the carriers, and over time. We further assume that $\alpha_{m}^{(k)}$ is Rayleigh distributed with pdf $f_{\alpha_{m}^{(k)}}(x)=2 x e^{-x^{2}}$, for $x \geq 0$, and $\phi_{m}^{(k)}$ is uniformly distributed over $(-\pi, \pi]$. The power spectral density (PSD) of the jammer, $S_{J}(f)$, is assumed to be of the following form [10]:

$$
S_{J}(f)= \begin{cases}\frac{\eta_{J}^{(l)}}{2} & \text { for } f_{J}^{(l)}-\frac{W_{J}^{(l)}}{2} \leq|f| \leq f_{J}^{(l)}+\frac{W_{J}^{(l)}}{2} \\ 0 & \text { otherwise }\end{cases}
$$

where, for $l=1, \ldots, L, \eta_{J}^{(l)}$ is the one-sided PSD of the jammer with a bandwidth of $W_{J}^{(l)}$ at the center frequency $f_{J}^{(l)}$.

Assuming perfect synchronization of carrier, code, and bit of the first user (i.e., the user of interest), the received signal (27) is first chip-matched filtered using the band-pass filters $H^{*}(f-$ $\left.f_{i}\right)+H^{*}\left(f+f_{i}\right), i=1, \ldots, L$, and then low-pass filtered with $\sqrt{2} \cos \left(2 \pi f_{i} t+\phi_{i}^{(1)}\right), i=1, \ldots, L$. Each of these $L$ outputs are correlated using the local pseudo-noise sequences. If $z_{i}$ denotes the output of the correlator on the $i$ th sub-carrier, then we have

$$
z_{i}=S_{i}+I_{i}+J_{i}+N_{i}
$$


where $S_{i}$ is the desired signal, $I_{i}$ is the signal due to the other $K_{u}-1$ interfering users, $J_{i}$ is the contribution due to the jammer and $N_{i}$ is the output due to AWGN. From [10], $I_{i}$ and $J_{i}$ are independent complex-Gaussian r.vs. From [11, Eqn. (23)], the mean of $z_{i}$, conditioned upon $\alpha_{i}^{(1)}$ and $d_{\lfloor n / N\rfloor}^{(1)}$, is

$$
E\left[z_{i} \mid \alpha_{i}^{(1)}, d_{\lfloor n / N\rfloor}^{(1)}\right]=d^{(1)} N \sqrt{E_{c}} \alpha_{i}^{(1)},
$$

where $d^{(1)}= \pm 1$ is the transmitted code symbol. To obtain the variance of $z_{i}$, conditioned on $\alpha_{i}^{(1)}$, we assume that the interference from other users, the PBI, and the AWGN are independent of each other. With this, we have

$$
\begin{aligned}
\operatorname{Var}\left\{z_{i} \mid \alpha_{i}^{(1)}\right\} \triangleq & \sigma_{i}^{2} \\
= & \operatorname{Var}\left\{I_{i} \mid \alpha_{i}^{(1)}\right\}+\operatorname{Var}\left\{J_{i} \mid \alpha_{i}^{(1)}\right\}+ \\
& \operatorname{Var}\left\{N_{i} \mid \alpha_{i}^{(1)}\right\} \\
\approx & N R_{I_{i}}(0)+N R_{J_{i}}(0)+N \eta_{0} / 2,
\end{aligned}
$$

where $R_{I_{i}}(\tau)$ and $R_{J_{i}}(\tau)$ are the autocorrelation functions of the interference and jammer, respectively. In (31), the approximation in the last step is due to ignoring the contribution of $R_{I_{i}}(\tau)$ and $R_{J_{i}}(\tau)$ when $\tau \neq 0$ [11, Eqns. (25)-(27)]. For simplicity, let $f_{J}^{(l)}=f_{l}$ and $W_{J}^{(l)}=W_{1}, \forall l=1, \ldots, L$. Then, with the help of [11]

$\sigma_{i}^{2}=\frac{N E_{c}\left(K_{u}-1\right)\left(1-\frac{\beta}{4}\right)}{2}+\frac{N \eta_{J}^{(i)}}{2}+\frac{N \eta_{0}}{2}, i=1, \ldots, L$.

Note that the total jammer power is given by $P_{J}=$ $\sum_{l=1}^{L} \eta_{J}^{(l)} W_{J}^{(l)}=\sum_{l=1}^{L} P_{J}^{(l)}$, where $P_{J}^{(l)}=\eta_{J}^{(l)} W_{J}^{(l)}$. The jammer-to-signal power ratio (JSR) is defined as

$$
\mathrm{JSR}=\frac{P_{J}}{E_{c} / T_{c}}=\sum_{l=1}^{L} \frac{\eta_{J}^{(l)} W_{J}^{(l)} T_{c}}{E_{c}}=\sum_{l=1}^{L} \mathrm{JSR}_{l},
$$

where $\mathrm{JSR}_{l}=\eta_{J}^{(l)} W_{J}^{(l)} /\left(E_{c} / T_{c}\right)=\eta_{J}^{(l)} W_{J}^{(l)} T_{c} / E_{c}$.

When the effective noise variances, $\left\{\sigma_{l}^{2}\right\}_{l=1}^{L}$, are known to the receiver, for each code symbol, the $L$ outputs, $z_{l}, l=$ $1, \ldots, L$, are processed using MRC to result in an output $Z_{\mathrm{MRC}}$. However, in the absence of $\left\{\sigma_{l}^{2}\right\}_{l=1}^{L}$, the receiver processing is termed as sub-optimum MRC (sMRC), and the output $Z$ is denoted by $Z_{\mathrm{sMRC}}$, and is given by

$$
Z_{\mathrm{sMRC}}=\sum_{l=1}^{L} \alpha_{l}^{(1)} z_{l}=d^{(1)} N \sqrt{E_{c}} \sum_{l=1}^{L}\left[\alpha_{l}^{(1)}\right]^{2}+\xi,
$$

where $\xi$ is a zero-mean Gaussian r.v with a conditional variance $\sigma_{\xi}^{2}=\sum_{l=1}^{L}\left(\alpha_{l}^{(1)} \sigma_{l}\right)^{2}$. The instantaneous SNR, $\gamma_{\mathrm{sMRC}}$, at the output of sMRC is

$$
\gamma_{\mathrm{sMRC}}=\frac{\left[\sum_{l=1}^{L}\left(\alpha_{l}^{(1)}\right)^{2}\right]^{2}}{\sum_{l=1}^{L} \frac{\left[\alpha_{l}^{(1)}\right]^{2}}{\bar{\gamma}_{l}}},
$$

where $\bar{\gamma}_{l}=N^{2} E_{c} /\left(2 \sigma_{l}^{2}\right)$ is the average signal-to-interferenceplus-noise ratio (SINR) on the $l$ th sub-carrier. Using (32), we simplify $\bar{\gamma}_{l}$ as

$$
\bar{\gamma}_{l}=\frac{1}{L} \times \frac{r_{c} \gamma_{b}}{1+\frac{r_{c}}{\mathcal{S}_{F}} \gamma_{b}\left(K_{u}-1\right)(1-\beta / 4)+\frac{r_{c}}{\mathcal{S}_{F}} \gamma_{b} \frac{\mathrm{JSR}_{l} L}{(1+\beta)}},
$$

where $l=1, \ldots, L, E_{b}=\mathcal{S}_{F} E_{c} / r_{c}$ and $\gamma_{b}=E_{b} / N_{0}$ is the SNR per information bit. For simplicity, let us define

$$
\bar{\gamma}=\frac{1}{L} \times \frac{r_{c} \gamma_{b}}{1+\frac{r_{c}}{\mathcal{S}_{F}} \gamma_{b}\left(K_{u}-1\right)(1-\beta / 4)}
$$

and, for $l=1, \ldots, L$,

$$
\bar{\rho}_{l}=\frac{1}{1+\beta} \times \frac{\mathrm{JSR}_{l} L \gamma_{b} r_{c} / \mathcal{S}_{F}}{1+\frac{r_{c}}{\mathcal{S}_{F}} \gamma_{b}\left(K_{u}-1\right)(1-\beta / 4)} .
$$

Eqn. (37) captures the average SINR in the absence of PBI, whereas (38) takes into account the jammer's contribution. Using (37) and (38), $\bar{\gamma}_{l}$ of (36) has the following compact form

$$
\bar{\gamma}_{l}=\frac{\bar{\gamma}}{1+\bar{\rho}_{l}} \triangleq \frac{1}{b_{l}} .
$$

When the receiver has perfect knowledge of $\left\{\sigma_{l}^{2}\right\}_{l=1}^{L}$, the SINR $\gamma_{\mathrm{MRC}}$ is given by [10]

$$
\gamma_{\mathrm{MRC}}=\sum_{l=1}^{L} \bar{\gamma}_{l}\left[\alpha_{l}^{(1)}\right]^{2}=\sum_{l=1}^{L} \frac{\bar{\gamma}}{1+\bar{\rho}_{l}}\left[\alpha_{l}^{(1)}\right]^{2} .
$$

We now derive the average pairwise error probability (PEP) with BPSK signaling. The probability that the transmitted codeword $\mathbf{x}=\left(x_{1}, x_{2}, \ldots, x_{N}\right), x_{i} \in\left\{-\sqrt{E_{s}},+\sqrt{E_{s}}\right\}$, is erroneously decoded as $\mathbf{y}=\left(y_{1}, y_{2}, \ldots, y_{N}\right), y_{i} \in$ $\left\{-\sqrt{E_{s}},+\sqrt{E_{s}}\right\}$, is given by (41), shown at the top of the next page, where $Q(x)=\int_{x}^{\infty} e^{-u^{2} / 2} d u / \sqrt{2 \pi}$. Let $\mathbf{x}$ and $\mathbf{y}$ differ in $d$ positions, $n_{1}, n_{2}, \ldots, n_{d}$. With this, (41) simplifies to [1]

$$
\begin{aligned}
& \operatorname{Prob}\left(d, \mathbf{n}^{(d)}\right)=E\left[Q\left(\frac{\sum_{l=1}^{L} \sum_{k=1}^{d} \alpha_{l}^{2}\left(n_{k}\right)}{\sqrt{\sum_{l=1}^{L} \sum_{k=1}^{d} \frac{\alpha_{l}^{2}\left(n_{k}\right)}{\bar{\gamma}_{l}\left(n_{k}\right)}}}\right)\right] \\
& =\frac{1}{\pi} \int_{0}^{\frac{\pi}{2}} \mathcal{L}_{\gamma_{\text {coded-MC-CDMA-PBI }}}\left(\frac{1}{2 \sin ^{2} \theta}\right) d \theta
\end{aligned}
$$

where $\mathbf{n}^{(d)}=\left(n_{1}, n_{2}, \ldots, n_{d}\right), \bar{\gamma}_{l}\left(n_{k}\right)=E_{s} /\left(2 \sigma_{l}^{2}\left(n_{k}\right)\right)$ is the average SINR on the $l$ sub-carrier for the $n_{k}$ th code symbol and

$$
\gamma_{\text {coded-MC-CDMA-PBI }}=\frac{\left[\sum_{l=1}^{L} \sum_{k=1}^{d} \alpha_{l}^{2}\left(n_{k}\right)\right]^{2}}{\sum_{l=1}^{L} \sum_{k=1}^{d} \frac{\alpha_{l}^{2}\left(n_{k}\right)}{\bar{\gamma}_{l}\left(n_{k}\right)}} .
$$

Once again, the uncoded as well as the coded performances of an MC-CDMA systems with PBI, characterized by the statistics of $\gamma_{\mathrm{sMRC}}$ in (35) and the PEP in (42), respectively, can be quantified with the help of (6)-(11). It is worth mentioning that unlike the Chernoff bound based average PEP in [9], [10], (42) presents an exact expression that is applicable to both optimum and sub-optimum receivers.

\section{RESUlTS AND Discussion}

In this section, we present some numerical and simulation results to illustrate the usefulness of our analytical results in Section II as applied to the systems exemplified in Section III.

The outage probability of MRC receiver output SNR is shown in Figs. 1 and 2, as a function of the outage threshold $\gamma_{T}$, with $L=4$ receive antennas. The average received SNR 


$$
\begin{aligned}
\operatorname{Prob}(\mathbf{x} \rightarrow \mathbf{y}) & =\operatorname{Prob}\left(\sum_{l=1}^{L} \sum_{n=1}^{N}\left(r_{l}(n)-\alpha_{l}(n) x_{n}\right)^{2}>\sum_{l=1}^{L} \sum_{n=1}^{N}\left(r_{l}(n)-\alpha_{l}(n) y_{n}\right)^{2}\right) \\
& =\operatorname{Prob}\left(\sum_{l=1}^{L} \sum_{n=1}^{N}\left(y_{n}-x_{n}\right) \eta_{l}(n) \alpha_{l}(n)>\sum_{l=1}^{L} \sum_{n=1}^{N}\left(x_{n}-y_{n}\right) x_{n} \alpha_{l}^{2}(n)\right) \\
& =E\left[Q\left(\frac{\sum_{l=1}^{L} \sum_{n=1}^{N} \alpha_{l}^{2}(n) x_{n}\left(x_{n}-y_{n}\right)}{\sqrt{\sum_{l=1}^{L} \sum_{n=1}^{N} \alpha_{l}^{2}(n) \sigma_{l}^{2}(n)\left(y_{n}-x_{n}\right)^{2}}}\right)\right] .
\end{aligned}
$$

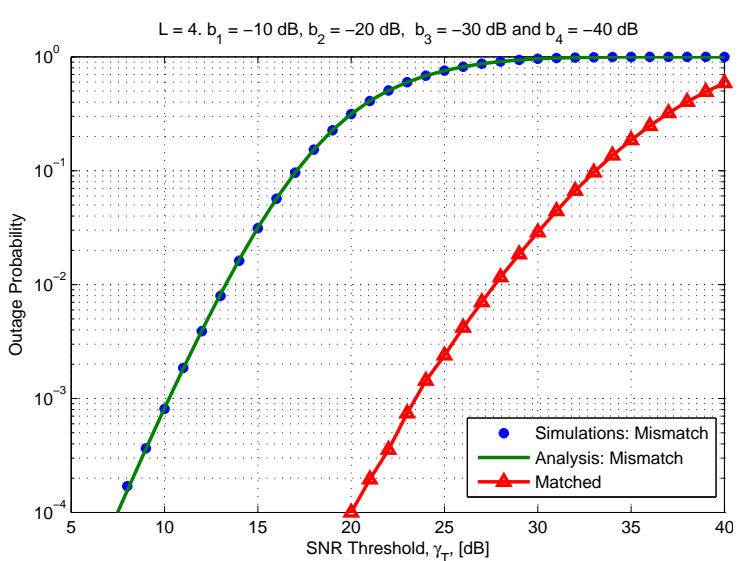

Fig. 1. The impact of unknown average noise power on the outage probability performance of the mismatched MRC receiver. The number of branches is $L=4$ with $\bar{\gamma}_{1}=10 \mathrm{~dB}, \bar{\gamma}_{2}=20 \mathrm{~dB}, \bar{\gamma}_{3}=30 \mathrm{~dB}$, and $\bar{\gamma}_{4}=40 \mathrm{~dB}$. For comparison purposes, outage performance of the ideal MRC (i.e., with knowledge of the average noise power) is also shown.

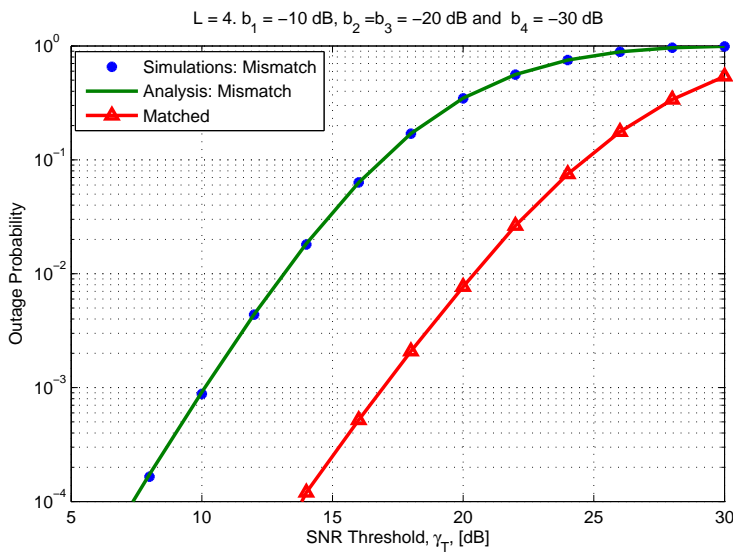

Fig. 2. Outage probability of MRC receiver with mismatched noise powers. The number of branches is $L=4$ with $\bar{\gamma}_{1}=10 \mathrm{~dB}, \bar{\gamma}_{2}=\bar{\gamma}_{3}=20 \mathrm{~dB}$, and $\bar{\gamma}_{4}=30 \mathrm{~dB}$. For comparison purposes, outage performance of the ideal MRC (i.e., with knowledge of the average noise power) is also shown.

per branch (in $\mathrm{dB}$ ) in Fig. 1 is set to $\bar{\gamma}_{1}=10, \bar{\gamma}_{2}=20$, $\bar{\gamma}_{3}=30$ and $\bar{\gamma}_{4}=40$, whereas they are set to $\bar{\gamma}_{1}=10$, $\bar{\gamma}_{2}=\bar{\gamma}_{3}=20$ and $\bar{\gamma}_{3}=30$ in Fig. 2. An excellent match between the analytical and simulation results is observed in Figs. 1 and 2. At an outage probability of $10^{-3}$, the noise variance-agnostic MRC receiver in Fig. 1 has a loss of about 13 $\mathrm{dB}$, whereas the loss is approximately $7 \mathrm{~dB}$ with the parameters chosen in Fig. 2.

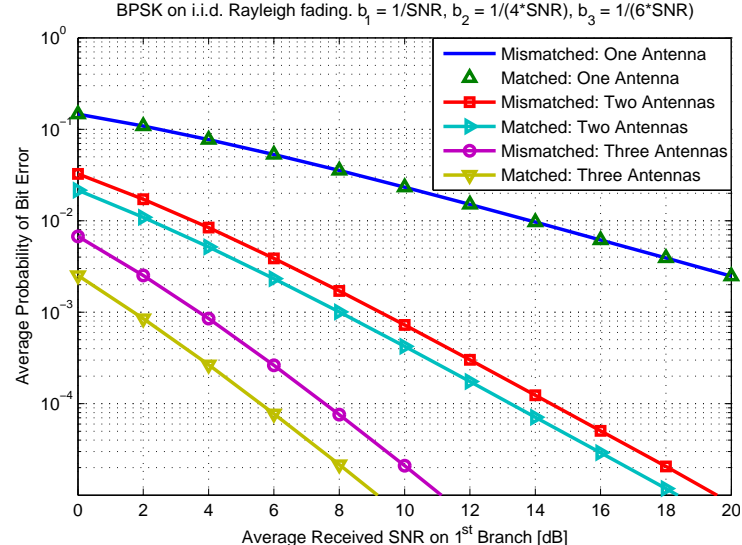

(a) BPSK Modulation

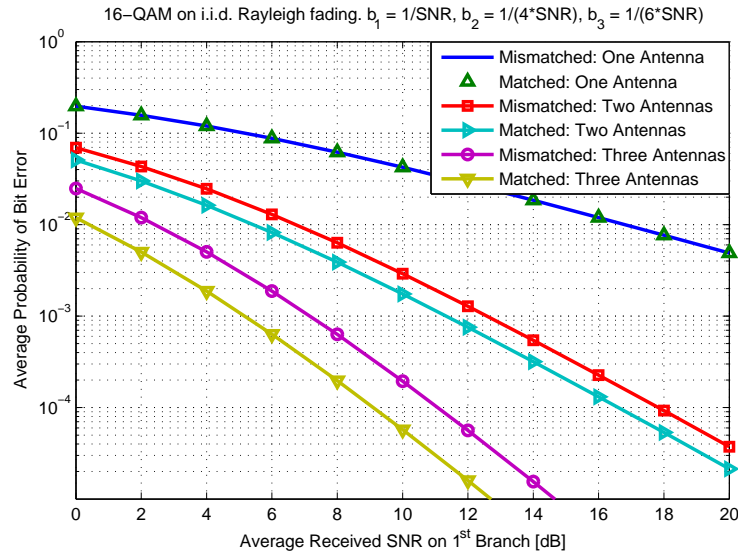

(b) 16-QAM Modulation

Fig. 3. Average BER of BPSK and 16-QAM modulations with mismatched MRC receiver. With up to $L=3$ antennas at the receiver, we set $b_{1}=\frac{1}{\gamma_{1}}$, $b_{2}=\frac{1}{4 \bar{\gamma}_{1}}$ and $b_{3}=\frac{1}{6 \bar{\gamma}_{1}}$, where $\bar{\gamma}_{1}$ is the average received SNR on the first branch.

In Fig. 3 the average probability of error of mismatched $\mathrm{MRC}$ receiver is compared against the ideal MRC receiver. In particular, Fig. 3(a) shows the error performance with BPSK modulation whereas Fig. 3(b) the performance with 16-QAM modulation. With up to 3 receiver antennas, we set $b_{1}=1 / \bar{\gamma}_{1}$, $b_{2}=1 /\left(4 \bar{\gamma}_{1}\right)$, and $b_{3}=1 /\left(6 \bar{\gamma}_{1}\right)$, where $\bar{\gamma}_{1}$ is the average received SNR on the first branch, and plot the error rates in Fig. 3 as a function of $\bar{\gamma}_{1}$. As expected, Figs. 3(a) and 3(b) confirm that knowledge of the noise variance is not required with single-antenna reception. However, at an error rate of $10^{-4}$, Figs. 3(a) and 3(b) reveal that lack of noise 
variance knowledge leads to a loss of approximately 1 and $2 \mathrm{~dB}$ respectively with two and three receive antennas.

The average symbol error rate of $M$-PSK modulation with diversity and imperfect CSI is shown in Fig. 4 with $L=3$ antennas and $M \in\{2,4,8\}$. In Fig. 4, we set $\rho_{1}^{2}=0.99$, $\rho_{2}^{2}=0.95, \rho_{3}^{2}=0.95$ and $\Omega_{l}=1, l=1, \ldots, 3$. With the constant $C=1$, we use $\Lambda_{l}$ that satisfies $\rho_{l} \sqrt{\Omega_{l} \Lambda_{l}}=$ C, $l=1, \ldots, 3$. Performance of the ideal MRC receiver that knows the knowledge of noise variances is contrasted against the mismatched MRC receiver that ignores them. Since the per-branch effective noise variance (from Section III-B), $N_{0}+E_{s}\left(1-\rho_{l}^{2}\right) \Omega_{l}, l=1, \ldots, L$, increases with the operating SNR, from Fig. 4 we observe that both the optimum and mismatched MRC receivers suffer from error floor. However, upon comparing the high SNR performance of mismatched and optimum receivers, we conclude from Fig. 4 that knowledge of noise variances is still beneficial to improve the error floor of mismatched receiver.

Finally, the PEP of a coded MC-CDMA system with PBI, described in Section III-C, is plotted in Fig. 5 as a function of the total average received SINR $\bar{\gamma}_{\text {Total }} \triangleq \sum_{k=1}^{L} \bar{\gamma} /\left(1+\bar{\rho}_{k}\right)$. Here, we consider $L=4$ sub-carriers and the distance between the two codewords of interest, $d$, is 2 . For simplicity, the system parameters in Section III-C are chosen in such a way that the average SINR on the sub-carrier $i, i=1, \ldots, L$, is equal to the $\frac{2 i}{L(L+1)}$ fraction of the total average SINR over the entire bandwidth. We also assume that on a given sub-carrier the two codewords at the differing positions have identical average SINRs. The PEP in Fig. 5 shows that the analysis, based on (42) and (11), matches excellently with the simulation results. At a PEP of $10^{-3}$, comparing the ideal performance in Fig. 5, we conclude that there is a loss of approximately $1.0 \mathrm{~dB}$ in SINR due to lack of knowledge of average interference power at the receiver.

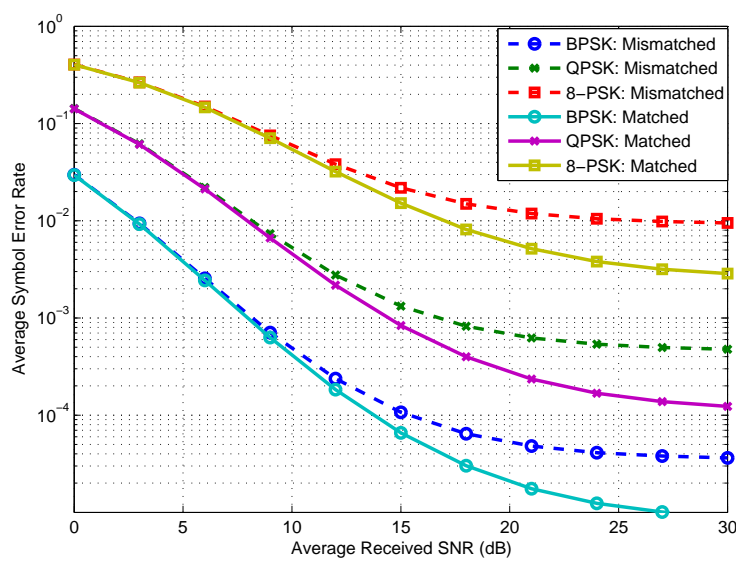

Fig. 4. Average symbol error rate of $M$-PSK modulation, $M \in\{2,4,8\}$, with $L=3$ diversity branches. Here, we set $\rho_{1}^{2}=0.99, \rho_{2}^{2}=0.95, \rho_{3}^{2}=0.9$ and $\mathrm{C}=\Omega_{l}=1, l=1, \ldots, L$. For comparison purposes, average symbol error performance of the ideal MRC (i.e., with knowledge of the effective average noise powers, $\left.N_{0}+E_{s}\left(1-\rho_{l}^{2}\right) \Omega_{l}, l=1, \ldots, L\right)$ is also shown.

\section{CONCLusion}

We presented the pdf, the cdf, and the LT of the pdf of the r.v $Z$ defined as $Z=\left(\sum_{j=1}^{L} X_{j}\right)^{2} /\left(\sum_{j=1}^{L} b_{j} X_{j}\right)$,

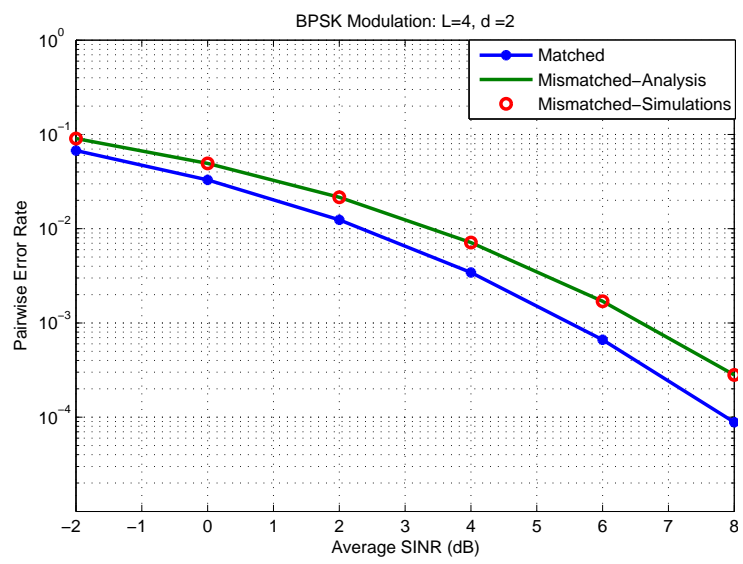

Fig. 5. Average pairwise error probability of coded MC-CDMA system with $L=4$ sub-carriers. The two codewords differ in $d=2$ positions. The system parameters in Section III-C are chosen such that the average SINR on the sub-carrier $i, i=1, \ldots, 4$, is equal to the $i / 10$ fraction of the total average SINR over the entire bandwidth. It is also assumed that on a given sub-carrier the two codewords at the differing positions have identical average SINRs.

where $X_{1}, X_{2}, \ldots, X_{L}$ are i.i.d exponential r.vs with unit mean and $b_{1}, b_{2}, \ldots, b_{L}$ are positive scalars. To illustrate the usefulness of this contribution we presented three application examples: $i$ ) impact of mismatched noise variances on MRC receiver performance $i$ ) $M$-ary PSK receiver performance with diversity and imperfect channel estimation, and iii) the performance of coded multi-carrier CDMA systems with an unknown narrow-band interference.

\section{REFERENCES}

[1] M. K. Simon and M.-S. Alouini, Digital Communications over Fading Channels: A Unified Approach to Performance Analysis, John Wiley \& Sons, Inc., 2004.

[2] M. Abramowitz and I. A. Stegun, Handbook of Mathematical Functions, Applied Mathematics Series 55. New York: National Bureau of Standard, 1964.

[3] Y-C. Ko and T. Luo, "Effect of noise imbalance on dual-MRC over Rayleigh fading channels, "IEEE Transacations on Wireless Communications, vol. 5, no. 3, March 2006, pp. 514-518.

[4] S. Manohar, V. Tikiya, R. Annavajjala and A. Chockalingam, "BERoptimal linear parallel interference cancellation for multicarrier DSCDMA in Rayleigh fading," IEEE Trans. Commun., vol. 55, no. 6, June 2007, pp. 1253-1265.

[5] R. Annavajjala, A. Chockalingam and S. K. Mohammed, "On a ratio of functions of exponential random variables and some applications." Technical Report, WRL-TR-2010-01, April 2010. Available at http://wrl. ece.iisc.ernet.in.

[6] L. Brenman, "Linear diversity combining schemes," Proc. IRE, 1956.

[7] R. Annavajjala, P. C. Cosman and L. B. Milstein, "Performance analysis of linear modulation schemes with generalized diversity combining on Rayleigh fading channels with noisy channel estimates," IEEE Trans. Info. Theory, vol. 53, no. 12, pp. 4701-4727, Dec. 2007.

[8] T. W. Anderson, An Introduction to Multivariate Statistical Analysis, Wiley Series in Probability and Statistics, 2003.

[9] D. N. Rowitch and L. B. Milstein, "Convolutionally coded multicarrier DS-CDMA systems in a multipath fading channel-Part I: Performance analysis," IEEE Trans. Commun., vol. 47, no. 10, pp. 1570-1582, October 1999.

[10] D. N. Rowitch and L. B. Milstein, "Convolutionally coded multicarrier DS-CDMA systems in a multipath fading channel-Part II: Narrow-band interference suppression," IEEE Trans. Commun., vol. 47, no. 11, pp. 1729-1736, November 1999.

[11] S. Kondo and L. B. Milstein, "On the performance of multicarrier DS CDMA systems," IEEE Trans. Commun., vol. 44, pp. 238-246, Februray 1996. 